\title{
The low-temperature heat capacity of some lanthanide zirconates
}

\author{
S. Lutique ${ }^{\mathrm{a}, 1}$, P. Javorskỳ ${ }^{\mathrm{a}, 2}$, R.J.M. Konings ${ }^{\mathrm{a}, *}$, J.-C. Krupa ${ }^{\mathrm{b}}$, A.C.G. van Genderen ${ }^{\mathrm{c}}$, \\ J.C. van Miltenburg ${ }^{\mathrm{c}}$, F. Wastin ${ }^{\mathrm{a}}$ \\ ${ }^{a}$ European Commission, Joint Research Center, Institute for Transuranium Elements, P.O. Box 2340, 76125 Karlsruhe, Germany \\ ${ }^{\mathrm{b}}$ Institut de Physique Nucléaire, CNRS-IN2P3, 91406 Orsay Cedex, France \\ ${ }^{\mathrm{c}}$ Chemical Thermodynamics Group, Utrecht University, Padualaan 8, 3584 CH, Utrecht, Netherlands
}

Received 29 January 2004; accepted 29 March 2004

Available online 8 May 2004

\begin{abstract}
The heat capacities of the lanthanide zirconates $\mathrm{Gd}_{2} \mathrm{Zr}_{2} \mathrm{O}_{7}$ and $\mathrm{Eu}_{2} \mathrm{Zr}_{2} \mathrm{O}_{7}$ with pyrochlore structure were measured by adiabatic calorimetry and the hybrid adiabatic relaxation method in the temperature range ( 0.3 to 400$) \mathrm{K}$. A thermal anomaly was observed for $\mathrm{Gd}_{2} \mathrm{Zr}_{2} \mathrm{O}_{7}$ below $T=15 \mathrm{~K}$. From the measurements the thermodynamic functions of $\mathrm{Gd}_{2} \mathrm{Zr}_{2} \mathrm{O}_{7}$ and $\mathrm{Eu}_{2} \mathrm{Zr}_{2} \mathrm{O}_{7}$ were determined. By combining our results with the heat capacity data of $\mathrm{La}_{2} \mathrm{Zr}_{2} \mathrm{O}_{7}$, the lattice component of the heat capacity and the entropy were derived for the lanthanide series $\mathrm{Ln}_{2} \mathrm{Zr}_{2} \mathrm{O}_{7}(\mathrm{Ln}=\mathrm{La}$ to $\mathrm{Gd})$. The Schottky contributions were derived for $\mathrm{Ce}_{2} \mathrm{Zr}_{2} \mathrm{O}_{7}$, $\mathrm{Nd}_{2} \mathrm{Zr}_{2} \mathrm{O}_{7}$ and $\mathrm{Eu}_{2} \mathrm{Zr}_{2} \mathrm{O}_{7}$ by subtracting the lattice component from the experimental data, and compared to the values calculated from the crystal field energy levels.
\end{abstract}

(c) 2004 Elsevier Ltd. All rights reserved.

Keywords: Heat capacity; Pyrochlore; Calorimetry

\section{Introduction}

Pyrochlore-type compounds with general formula $\mathrm{A}_{2} \mathrm{~B}_{2} \mathrm{O}_{7}$, and especially zirconates $(\mathrm{B}=\mathrm{Zr})$, are materials with high scientific and technological importance. Recently, they have attracted attention in the scientific world as geometrically frustrated magnetic systems, exhibiting 'spin ice' behaviour [1-3]. During the last decades they have found many interesting industrial applications: they are studied for thermal barrier coating [4-7], sub-catalysts for automotive exhaust gases $\left(\mathrm{Ce}_{2} \mathrm{Zr}_{2} \mathrm{O}_{7+y}\right)$ [8-11] as well as for nuclear applications [12-21]. For the latter, which is the topic of our research, it is important that the chemical configuration of the

\footnotetext{
${ }^{*}$ Corresponding author. Fax: +49-7247-951-591.

E-mail address: konings@itu.fzk.de (R.J.M. Konings).

${ }^{1}$ Current address: Commissariat à l'Energie Atomique, Centre de Cadarache, DEN/DEC/SPUA/LTEC, 13108 St. Paul lez Durance, France.

${ }^{2}$ Permanent address: Department of Electronic Structures, Charles University, Ke Karlovu, 12116 Prague, Czech Republic.
}

lanthanide zirconate pyrochlores enables incorporation of actinides in the crystal lattice. This makes them potential candidates as inert matrix for the transmutation of actinides or as waste form for their storage.

Knowledge of the thermodynamic stability of these compounds is necessary for all industrial applications in order to determine their durability under typical process conditions. Until now only few thermodynamic studies have been reported for lanthanide zirconate pyrochlore compounds. The molar enthalpy of formation of some lanthanide zirconates $\mathrm{Ln}_{2} \mathrm{Zr}_{2} \mathrm{O}_{7}$ were reported for $\mathrm{Ln}=\mathrm{La}, \mathrm{Pr}, \mathrm{Nd}$ and $\mathrm{Sm}$ by Korneev et al. [22], for $\mathrm{Ln}=\mathrm{La}$ by Bolech et al. [23-25] and for $\mathrm{Ln}=\mathrm{Gd}$ by Helean et al. [26]. The heat capacity of some lanthanide zirconate was reported by Bolech et al. $[24,25](\mathrm{Ln}=\mathrm{La}$ and $\mathrm{Ce}$ ) in the temperature range ( 5 to 900$) \mathrm{K}$ and Lutique et al. $[16,27,28](\mathrm{Ln}=\mathrm{Nd})$ in the temperature range $(0.4$ to 1600$) \mathrm{K}$. In the present study, we extend the heat capacity data with $\mathrm{Gd}_{2} \mathrm{Zr}_{2} \mathrm{O}_{7}$ and $\mathrm{Eu}_{2} \mathrm{Zr}_{2} \mathrm{O}_{7}$, the results of which enable us to determine the lattice and excess components in the series $\mathrm{Ln}=\mathrm{La}$ to $\mathrm{Gd}$. 
TABLE 1

Experimental heat capacity $C_{\mathrm{p}, \mathrm{m}}^{\circ}$ for $\mathrm{Gd}_{2} \mathrm{Zr}_{2} \mathrm{O}_{7}$ obtained with the adiabatic calorimeter at temperature $T$

\begin{tabular}{|c|c|c|c|c|c|c|c|}
\hline \multirow[t]{2}{*}{$T / \mathrm{K}$} & $\underline{C_{\mathrm{p}, \mathrm{m}}^{\circ}}$ & $T / \mathrm{K}$ & $\underline{C_{\mathrm{p}, \mathrm{m}}^{\circ}}$ & $T / \mathrm{K}$ & $\underline{C_{\mathrm{p}, \mathrm{m}}^{\circ}}$ & $T / \mathrm{K}$ & $\underline{C_{\mathrm{p}, \mathrm{m}}^{\circ}}$ \\
\hline & \multicolumn{2}{|l|}{$\left(\mathrm{J} \cdot \mathrm{K}^{-1} \cdot \mathrm{mol}^{-1}\right)$} & \multicolumn{2}{|l|}{$\overline{\left(\mathrm{J} \cdot \mathrm{K}^{-1} \cdot \mathrm{mol}^{-1}\right)}$} & \multicolumn{2}{|l|}{$\left(\mathrm{J} \cdot \mathrm{K}^{-1} \cdot \mathrm{mol}^{-1}\right)$} & $\left(\mathrm{J} \cdot \mathrm{K}^{-1} \cdot \mathrm{mol}^{-1}\right)$ \\
\hline \multicolumn{8}{|c|}{ Run 1} \\
\hline 299.59 & 221.9 & 326.46 & 230.9 & 353.30 & 238.6 & 380.24 & 245.3 \\
\hline 302.61 & 222.9 & 329.45 & 231.7 & 356.29 & 239.4 & 383.24 & 246.0 \\
\hline 305.58 & 223.9 & 332.43 & 232.7 & 359.28 & 240.1 & 386.24 & 246.6 \\
\hline 308.56 & 225.1 & 335.41 & 233.6 & 362.27 & 240.8 & 389.24 & 247.3 \\
\hline 311.54 & 226.1 & 338.38 & 234.4 & 365.26 & 241.7 & 392.24 & 247.8 \\
\hline 314.52 & 227.1 & 341.37 & 235.3 & 368.26 & 242.3 & 395.35 & 248.4 \\
\hline 317.51 & 228.1 & 344.35 & 236.2 & 371.25 & 243.1 & 398.26 & 249.1 \\
\hline 320.49 & 229.0 & 347.33 & 237.0 & 374.24 & 243.9 & 401.27 & 249.9 \\
\hline 323.48 & 229.9 & 350.32 & 237.8 & 377.24 & 244.6 & 404.28 & 250.4 \\
\hline \multicolumn{8}{|c|}{ Run 2} \\
\hline 99.99 & 90.29 & 136.59 & 127.7 & 174.64 & 159.9 & 213.00 & 185.4 \\
\hline 101.94 & 92.84 & 139.50 & 130.4 & 177.58 & 162.1 & 215.96 & 187.0 \\
\hline 104.84 & 95.74 & 142.42 & 133.1 & 180.52 & 164.3 & 218.92 & 188.5 \\
\hline 107.71 & 99.12 & 145.33 & 135.8 & 183.47 & 166.4 & 221.88 & 190.2 \\
\hline 110.58 & 102.0 & 148.25 & 138.4 & 186.41 & 168.5 & 224.84 & 191.8 \\
\hline 113.46 & 105.0 & 151.18 & 140.9 & 189.36 & 170.6 & 227.80 & 193.4 \\
\hline 116.33 & 107.8 & 154.11 & 143.4 & 192.31 & 172.7 & 230.77 & 194.9 \\
\hline 119.22 & 110.5 & 157.03 & 145.8 & 195.26 & 174.7 & 233.73 & 196.4 \\
\hline 122.10 & 113.4 & 159.96 & 148.3 & 198.21 & 176.7 & 236.70 & 197.9 \\
\hline 125.00 & 116.6 & 162.89 & 150.6 & 201.17 & 178.5 & 239.67 & 199.4 \\
\hline 127.89 & 119.4 & 165.82 & 153.0 & 204.12 & 180.4 & 242.63 & 200.8 \\
\hline 130.78 & 122.3 & 168.76 & 155.4 & 207.08 & 182.2 & 245.60 & 202.1 \\
\hline 133.69 & 125.0 & 171.69 & 157.6 & 210.04 & 183.8 & 248.57 & 203.6 \\
\hline \multicolumn{8}{|c|}{ Run 3} \\
\hline 255.64 & 206.1 & 267.53 & 211.4 & 279.42 & 216.1 & 288.36 & 219.3 \\
\hline 258.62 & 207.6 & 270.50 & 212.6 & 282.40 & 217.2 & 291.34 & 220.2 \\
\hline 261.60 & 208.9 & 273.47 & 213.8 & 285.38 & 218.3 & 294.32 & 220.8 \\
\hline 264.56 & 210.1 & 276.45 & 215.0 & & & & \\
\hline \multicolumn{8}{|c|}{ Run 4} \\
\hline 298.71 & 220.9 & 327.34 & 230.5 & 357.18 & 239.0 & 387.12 & 246.2 \\
\hline 300.49 & 221.6 & 330.32 & 231.4 & 360.17 & 239.7 & 390.13 & 246.7 \\
\hline 303.49 & 222.4 & 333.30 & 232.3 & 363.16 & 240.5 & 393.14 & 247.2 \\
\hline 306.48 & 223.5 & 336.29 & 233.1 & 366.15 & 241.2 & 396.14 & 247.8 \\
\hline 309.46 & 224.6 & 339.27 & 234.0 & 369.14 & 242.0 & 399.15 & 248.5 \\
\hline 312.44 & 225.6 & 342.25 & 234.8 & 372.13 & 242.6 & 402.16 & 249.0 \\
\hline 315.42 & 226.6 & 345.23 & 235.7 & 375.12 & 243.5 & 405.18 & 249.7 \\
\hline 318.40 & 227.6 & 348.22 & 236.6 & 378.12 & 244.1 & & \\
\hline 321.38 & 228.6 & 351.21 & 237.4 & 381.12 & 244.8 & & \\
\hline 324.36 & 229.6 & 354.19 & 238.1 & 384.12 & 245.4 & & \\
\hline \multicolumn{8}{|c|}{ Run 5} \\
\hline 5.02 & 4.762 & 6.66 & 3.576 & 7.27 & 3.201 & 8.15 & 4.021 \\
\hline 5.39 & 4.085 & 6.69 & 3.439 & 7.78 & 3.179 & & \\
\hline \multicolumn{8}{|c|}{ Run 6} \\
\hline 5.75 & 4.144 & 16.10 & 2.529 & 21.67 & 5.071 & 25.75 & 8.184 \\
\hline 8.61 & 2.534 & 17.67 & 3.056 & 23.02 & 5.773 & 27.16 & 9.130 \\
\hline 11.57 & 2.026 & 19.00 & 3.599 & 24.36 & 6.997 & 28.60 & 10.54 \\
\hline \multicolumn{8}{|c|}{ Run 7} \\
\hline 5.15 & 4.003 & 13.82 & 2.004 & 19.81 & 3.798 & 25.48 & 7.856 \\
\hline 6.88 & 3.124 & 15.47 & 2.281 & 21.25 & 4.715 & & \\
\hline 9.34 & 2.281 & 16.94 & 3.015 & 22.65 & 5.561 & & \\
\hline 11.78 & 1.875 & 18.37 & 3.309 & 24.06 & 6.716 & & \\
\hline \multicolumn{8}{|c|}{ Run 8} \\
\hline 27.34 & 8.888 & 41.73 & 23.57 & 57.60 & 42.08 & 74.32 & 61.44 \\
\hline 28.59 & 10.17 & 43.44 & 25.54 & 59.42 & 44.18 & 76.21 & 63.60 \\
\hline 30.28 & 11.70 & 45.16 & 27.54 & 61.25 & 46.31 & 78.11 & 65.77 \\
\hline 31.85 & 13.20 & 46.90 & 29.50 & 63.10 & 48.49 & 80.01 & 67.99 \\
\hline
\end{tabular}


TABLE 1 (continued)

\begin{tabular}{|c|c|c|c|c|c|c|c|}
\hline \multirow[t]{2}{*}{$T / \mathrm{K}$} & $C_{\mathrm{p}, \mathrm{m}}^{\circ}$ & $T / \mathrm{K}$ & $C_{\mathrm{p}, \mathrm{m}}^{\circ}$ & $T / \mathrm{K}$ & $C_{\mathrm{p}, \mathrm{m}}^{\circ}$ & $T / \mathrm{K}$ & $C_{\mathrm{p}, \mathrm{m}}^{\circ}$ \\
\hline & \multicolumn{2}{|l|}{$\left(\mathrm{J} \cdot \mathrm{K}^{-1} \cdot \mathrm{mol}^{-1}\right)$} & \multicolumn{2}{|c|}{$\overline{\left(\mathrm{J} \cdot \mathrm{K}^{-1} \cdot \mathrm{mol}^{-1}\right)}$} & \multicolumn{2}{|c|}{$\overline{\left(\mathrm{J} \cdot \mathrm{K}^{-1} \cdot \mathrm{mol}^{-1}\right)}$} & $\left(\mathrm{J} \cdot \mathrm{K}^{-1} \cdot \mathrm{mol}^{-1}\right)$ \\
\hline 33.42 & 14.96 & 48.65 & 31.63 & 64.95 & 50.64 & 81.92 & 70.16 \\
\hline 35.03 & 16.67 & 50.41 & 33.67 & 66.81 & 52.75 & 83.83 & 72.04 \\
\hline 36.66 & 18.43 & 52.19 & 35.74 & 68.68 & 54.94 & 85.75 & 74.43 \\
\hline 38.33 & 19.78 & 53.98 & 37.79 & 70.55 & 57.09 & 87.67 & 76.62 \\
\hline 40.03 & 21.65 & 55.79 & 39.92 & 72.43 & 59.27 & 89.59 & 78.86 \\
\hline \multicolumn{8}{|c|}{ Run 9} \\
\hline 5.39 & 5.557 & 9.23 & 2.338 & 12.38 & 1.905 & 14.47 & 2.146 \\
\hline 6.39 & 3.337 & & & & & & \\
\hline \multicolumn{8}{|c|}{ Run 10} \\
\hline 6.57 & 4.653 & 10.62 & 1.859 & 14.41 & 2.054 & 17.71 & 2.843 \\
\hline 7.09 & 1.869 & 11.91 & 1.932 & 15.56 & 2.548 & 18.74 & 3.372 \\
\hline 8.75 & 2.305 & 13.18 & 2.063 & 16.65 & 2.604 & 19.70 & 3.988 \\
\hline \multicolumn{8}{|c|}{ Run 11} \\
\hline 21.15 & 4.222 & 34.71 & 16.30 & 51.01 & 34.30 & 68.78 & 55.04 \\
\hline 22.44 & 5.562 & 36.63 & 18.41 & 53.16 & 36.84 & 71.07 & 57.68 \\
\hline 23.86 & 6.517 & 38.60 & 20.08 & 55.33 & 39.42 & 73.38 & 60.34 \\
\hline 25.61 & 7.937 & 40.62 & 22.35 & 57.53 & 41.95 & 75.70 & 62.97 \\
\hline 27.35 & 9.282 & 42.65 & 24.76 & 59.74 & 44.49 & 78.02 & 65.59 \\
\hline 29.15 & 10.86 & 44.69 & 27.04 & 61.98 & 47.15 & 80.36 & 68.33 \\
\hline 30.99 & 12.58 & 46.77 & 29.41 & 64.23 & 49.75 & & \\
\hline 32.83 & 14.42 & 48.88 & 31.86 & 66.50 & 52.35 & & \\
\hline \multicolumn{8}{|c|}{ Run 12} \\
\hline 84.26 & 73.08 & 127.42 & 118.9 & 171.42 & 157.6 & 215.77 & 187.1 \\
\hline 86.68 & 75.97 & 129.86 & 121.3 & 173.88 & 159.3 & 218.24 & 187.9 \\
\hline 89.04 & 78.05 & 132.29 & 123.7 & 176.34 & 161.2 & 220.70 & 189.4 \\
\hline 91.40 & 80.73 & 134.72 & 125.9 & 178.80 & 163.0 & 223.17 & 190.4 \\
\hline 93.76 & 83.52 & 137.16 & 128.2 & 181.26 & 164.8 & 225.64 & 192.2 \\
\hline 96.13 & 85.94 & 139.59 & 130.2 & 183.72 & 166.4 & 228.11 & 193.4 \\
\hline 98.51 & 88.81 & 142.03 & 132.8 & 186.18 & 168.2 & 230.58 & 194.8 \\
\hline 100.90 & 91.06 & 144.47 & 134.8 & 188.64 & 169.8 & 233.05 & 195.7 \\
\hline 103.28 & 93.88 & 146.91 & 136.9 & 191.10 & 171.8 & 235.53 & 197.1 \\
\hline 105.68 & 96.49 & 149.35 & 139.3 & 193.56 & 173.3 & 238.00 & 198.4 \\
\hline 108.08 & 99.44 & 151.80 & 141.2 & 196.03 & 175.1 & 240.47 & 199.4 \\
\hline 110.48 & 101.8 & 154.25 & 143.3 & 198.49 & 176.5 & 242.94 & 200.6 \\
\hline 112.89 & 104.4 & 156.70 & 145.3 & 200.95 & 178.5 & 245.41 & 201.8 \\
\hline 115.30 & 106.7 & 159.15 & 147.6 & 203.42 & 179.6 & 247.88 & 203.0 \\
\hline 117.72 & 109.3 & 161.60 & 149.4 & 205.89 & 181.7 & 250.35 & 203.7 \\
\hline 120.14 & 110.9 & 164.06 & 151.7 & 208.35 & 183.1 & & \\
\hline 122.56 & 113.9 & 166.52 & 153.4 & 210.82 & 184.4 & & \\
\hline 124.99 & 116.5 & 168.97 & 155.4 & 213.29 & 185.7 & & \\
\hline
\end{tabular}

\section{Experimental}

Adiabatic calorimetry was used to measure the heat capacity on powders in the temperature range ( 3 to 400 ) $\mathrm{K}$. The calorimeter CAL V (Utrecht University) and its calibration were described previously [29]. Samples were prepared by co-precipitation from a solution of zirconium oxi-chloride $\mathrm{ZrOCl}_{2} \cdot 8 \mathrm{H}_{2} \mathrm{O}$ and lanthanide nitrate $\mathrm{Ln}\left(\mathrm{NO}_{3}\right)_{3} \cdot x \mathrm{H}_{2} \mathrm{O}$ with the right concentration using ammonia buffered to $\mathrm{pH} 11$. Powders were sintered in air: 72 $\mathrm{h}$ at $T=1723 \mathrm{~K}$ for $\mathrm{Gd}_{2} \mathrm{Zr}_{2} \mathrm{O}_{7}$, and $8 \mathrm{~h}$ at $T=1773 \mathrm{~K}$ for $\mathrm{Eu}_{2} \mathrm{Zr}_{2} \mathrm{O}_{7}$. X-ray diffraction revealed only pyrochlore phases with lattice parameters of (1.051 and 1.057) nm, respectively. Specimens with weight of $17.1 \mathrm{~g}$ for
$\mathrm{Gd}_{2} \mathrm{Zr}_{2} \mathrm{O}_{7}$ and $11.2 \mathrm{~g}$ for $\mathrm{Eu}_{2} \mathrm{Zr}_{2} \mathrm{O}_{7}$ were used. The experimental results are summarised in tables 1 and 2 .

The heat capacity was also measured by an hybrid adiabatic relaxation method using a PPMS instrument (Quantum Design) on polycrystalline sample (pellet piece) in the temperature range ( 0.36 to 29$) \mathrm{K}$. The accuracy of the PPMS instrument was verified by measuring a gold standard. The PPMS software used the two-tau relaxation method to determine the heat capacity values. $\mathrm{Gd}_{2} \mathrm{Zr}_{2} \mathrm{O}_{7}$ pellets were obtained by pressing beads that were prepared by sol-gel method and subsequently calcined. The pellets were sintered in air for $72 \mathrm{~h}$ at $T=1723 \mathrm{~K} . \mathrm{Eu}_{2} \mathrm{Zr}_{2} \mathrm{O}_{7}$ pellets were obtained by pressing co-precipitated powder after 
TABLE 2

Experimental heat capacity $C_{\mathrm{p}, \mathrm{m}}^{\circ}$ data for $\mathrm{Eu}_{2} \mathrm{Zr}_{2} \mathrm{O}_{7}$ obtained with the adiabatic calorimeter at temperature $T$

\begin{tabular}{|c|c|c|c|c|c|c|c|}
\hline \multirow[t]{2}{*}{$T / \mathrm{K}$} & $\underline{C_{\mathrm{p}, \mathrm{m}}^{\circ}}$ & $T / \mathrm{K}$ & $\underline{C_{\mathrm{p}, \mathrm{m}}^{\circ}}$ & $T / \mathrm{K}$ & $\underline{C_{\mathrm{p}, \mathrm{m}}^{\circ}}$ & $T / \mathrm{K}$ & $\underline{C_{\mathrm{p}, \mathrm{m}}^{\circ}}$ \\
\hline & \multicolumn{2}{|l|}{$\overline{\left(\mathrm{J} \cdot \mathrm{K}^{-1} \cdot \mathrm{mol}^{-1}\right)}$} & \multicolumn{2}{|l|}{$\overline{\left(\mathrm{J} \cdot \mathrm{K}^{-1} \cdot \mathrm{mol}^{-1}\right)}$} & \multicolumn{2}{|l|}{$\overline{\left(\mathrm{J} \cdot \mathrm{K}^{-1} \cdot \mathrm{mol}^{-1}\right)}$} & $\left(\mathrm{J} \cdot \mathrm{K}^{-1} \cdot \mathrm{mol}^{-1}\right)$ \\
\hline \multicolumn{8}{|c|}{ Run 1} \\
\hline 296.77 & 234.2 & 322.71 & 242.5 & 349.52 & 249.8 & 376.44 & 256.4 \\
\hline 298.86 & 235.4 & 325.68 & 243.3 & 352.51 & 250.5 & 379.44 & 257.1 \\
\hline 301.85 & 236.2 & 328.66 & 244.2 & 355.49 & 251.3 & 382.44 & 257.9 \\
\hline 304.83 & 237.1 & 331.64 & 244.8 & 358.48 & 252.2 & 385.45 & 258.5 \\
\hline 307.81 & 238.1 & 334.62 & 245.7 & 361.47 & 252.8 & 388.45 & 259.3 \\
\hline 310.79 & 239.0 & 337.60 & 246.6 & 364.46 & 253.4 & 391.46 & 259.8 \\
\hline 313.77 & 239.7 & 340.58 & 247.4 & 367.45 & 254.3 & 394.47 & 260.4 \\
\hline 316.75 & 240.5 & 343.56 & 248.0 & 370.44 & 255.0 & 397.48 & 260.9 \\
\hline 319.73 & 241.4 & 346.54 & 249.0 & 373.44 & 255.7 & 400.50 & 261.5 \\
\hline \multicolumn{8}{|c|}{ Run 2} \\
\hline 6.21 & 0.125 & 14.77 & 1.21 & 20.77 & 4.20 & 27.57 & 9.32 \\
\hline 9.55 & 0.275 & 15.90 & 1.34 & 22.11 & 5.03 & 28.93 & 10.85 \\
\hline 11.31 & 0.422 & 17.07 & 2.01 & 23.45 & 6.06 & & \\
\hline 12.64 & 0.611 & 18.30 & 2.78 & 24.79 & 7.18 & & \\
\hline 13.66 & 0.927 & 19.51 & 3.50 & 26.16 & 8.72 & & \\
\hline \multicolumn{8}{|c|}{ Run 3} \\
\hline 5.72 & 0.213 & 11.76 & 0.597 & 16.59 & 2.01 & 21.59 & 4.63 \\
\hline 7.23 & 0.151 & 13.58 & 1.03 & 18.23 & 2.65 & 23.34 & 5.89 \\
\hline 9.16 & 0.249 & 15.08 & 1.43 & 19.90 & 3.67 & 25.15 & 7.66 \\
\hline \multicolumn{8}{|c|}{ Run 4} \\
\hline 28.09 & 10.36 & 44.74 & 29.19 & 64.09 & 55.21 & 84.86 & 82.78 \\
\hline 29.66 & 11.71 & 46.78 & 31.88 & 66.34 & 58.22 & 87.22 & 85.79 \\
\hline 31.42 & 13.49 & 48.86 & 34.70 & 68.62 & 61.34 & 89.59 & 88.77 \\
\hline 33.19 & 15.44 & 50.97 & 37.42 & 70.90 & 64.43 & 91.96 & 91.74 \\
\hline 34.98 & 17.42 & 53.10 & 40.28 & 73.20 & 67.49 & 94.34 & 94.65 \\
\hline 36.84 & 19.72 & 55.25 & 43.15 & 75.51 & 70.56 & 96.73 & 97.58 \\
\hline 38.76 & 21.63 & 57.43 & 46.15 & 77.84 & 73.59 & 99.12 & 100.5 \\
\hline 40.73 & 24.05 & 59.63 & 49.18 & 80.17 & 76.66 & & \\
\hline 42.72 & 26.66 & 61.85 & 52.17 & 82.51 & 79.73 & & \\
\hline \multicolumn{8}{|c|}{ Run 5} \\
\hline 98.55 & 99.47 & 124.07 & 128.9 & 150.96 & 154.7 & 178.01 & 176.7 \\
\hline 99.88 & 101.4 & 126.50 & 131.3 & 153.42 & 156.9 & 180.47 & 178.5 \\
\hline 102.32 & 104.7 & 128.94 & 133.9 & 155.88 & 159.0 & 182.93 & 180.3 \\
\hline 104.73 & 107.3 & 131.38 & 136.4 & 158.33 & 161.1 & 185.40 & 182.1 \\
\hline 107.13 & 110.5 & 133.82 & 138.8 & 160.79 & 163.1 & 187.86 & 183.9 \\
\hline 109.54 & 113.3 & 136.26 & 141.1 & 163.26 & 165.1 & 190.32 & 185.6 \\
\hline 111.95 & 115.8 & 138.71 & 143.5 & 165.71 & 167.2 & 192.79 & 187.4 \\
\hline 114.37 & 118.4 & 141.16 & 145.8 & 168.17 & 169.2 & 195.26 & 189.0 \\
\hline 116.79 & 121.0 & 143.61 & 148.0 & 170.63 & 171.1 & 197.72 & 190.6 \\
\hline 119.21 & 123.4 & 146.06 & 150.3 & 173.09 & 172.9 & & \\
\hline 121.64 & 125.7 & 148.51 & 152.6 & 175.55 & 174.8 & & \\
\hline \multicolumn{8}{|c|}{ Run 6} \\
\hline 197.47 & 190.2 & 229.62 & 207.8 & 261.88 & 221.9 & 294.25 & 234.0 \\
\hline 199.92 & 191.7 & 232.10 & 209.0 & 264.37 & 222.9 & 296.75 & 234.9 \\
\hline 202.41 & 193.3 & 234.58 & 210.2 & 266.86 & 224.0 & 299.25 & 235.6 \\
\hline 204.87 & 194.9 & 237.06 & 211.5 & 269.34 & 224.9 & 301.75 & 236.3 \\
\hline 209.81 & 197.4 & 242.02 & 213.7 & 274.32 & 226.9 & 306.74 & 238.2 \\
\hline 212.29 & 198.8 & 244.50 & 214.9 & 276.80 & 227.7 & 309.23 & 239.0 \\
\hline 214.76 & 200.2 & 246.98 & 216.0 & 279.29 & 228.6 & 311.73 & 239.6 \\
\hline 217.24 & 201.5 & 249.46 & 217.0 & 281.78 & 229.5 & 314.23 & 240.4 \\
\hline 219.71 & 202.8 & 251.94 & 218.3 & 284.28 & 230.5 & 316.73 & 241.1 \\
\hline 222.19 & 204.0 & 254.43 & 218.6 & 286.77 & 231.3 & 319.23 & 241.8 \\
\hline 224.67 & 205.3 & 256.91 & 219.7 & 289.26 & 231.5 & & \\
\hline 227.15 & 206.5 & 259.40 & 220.8 & 291.76 & 233.1 & & \\
\hline \multicolumn{8}{|c|}{ Run 7} \\
\hline 299.12 & 235.8 & 326.45 & 244.1 & 353.73 & 251.7 & 381.10 & 258.6 \\
\hline 301.64 & 236.7 & 328.93 & 244.8 & 356.21 & 252.3 & 383.59 & 259.2 \\
\hline
\end{tabular}


TABLE 2 (continued)

\begin{tabular}{|c|c|c|c|c|c|c|c|}
\hline \multirow[t]{2}{*}{$T / \mathrm{K}$} & $C_{\mathrm{p}, \mathrm{m}}^{\circ}$ & \multirow[t]{2}{*}{$T / \mathrm{K}$} & $C_{\mathrm{p}, \mathrm{m}}^{\circ}$ & \multirow[t]{2}{*}{$T / \mathrm{K}$} & $C_{\mathrm{p}, \mathrm{m}}^{\circ}$ & \multirow[t]{2}{*}{$T / \mathrm{K}$} & \multirow{2}{*}{$\frac{C_{\mathrm{p}, \mathrm{m}}^{\circ}}{\left(\mathrm{J} \cdot \mathrm{K}^{-1} \cdot \mathrm{mol}^{-1}\right)}$} \\
\hline & $\left(\mathrm{J} \cdot \mathrm{K}^{-1} \cdot \mathrm{mol}^{-1}\right)$ & & $\left(\mathrm{J} \cdot \mathrm{K}^{-1} \cdot \mathrm{mol}^{-1}\right)$ & & $\left(\mathrm{J} \cdot \mathrm{K}^{-1} \cdot \mathrm{mol}^{-1}\right)$ & & \\
\hline 304.12 & 237.3 & 331.41 & 245.6 & 358.70 & 253.0 & 386.09 & 259.7 \\
\hline 306.61 & 238.1 & 333.89 & 246.3 & 361.18 & 253.7 & 388.59 & 260.3 \\
\hline 309.10 & 239.0 & 336.37 & 247.0 & 363.66 & 254.2 & 391.09 & 260.5 \\
\hline 311.58 & 239.5 & 338.85 & 247.7 & 366.15 & 254.9 & 393.59 & 261.2 \\
\hline 314.06 & 240.4 & 341.33 & 248.3 & 368.64 & 255.5 & 396.09 & 262.5 \\
\hline 316.54 & 240.9 & 343.81 & 249.0 & 371.13 & 256.1 & 398.60 & 263.0 \\
\hline 319.02 & 241.6 & 346.29 & 249.8 & 373.62 & 256.7 & 401.11 & 262.9 \\
\hline 321.50 & 242.6 & 348.77 & 250.4 & 376.11 & 257.3 & & \\
\hline 323.97 & 243.4 & 351.25 & 251.0 & 378.61 & 257.9 & & \\
\hline
\end{tabular}

compaction/milling steps and sintering in air for $2 \mathrm{~h}$ at $T=(1773$ and 1473) K. Pyrochlore phases were observed and lattice parameters of (1.055 and 1.0574) nm were measured for $\mathrm{Gd}_{2} \mathrm{Zr}_{2} \mathrm{O}_{7}$ and $\mathrm{Eu}_{2} \mathrm{Zr}_{2} \mathrm{O}_{7}$, respectively. The experimental data from the PPMS measurements are summarised elsewhere [27] and are available upon request from the authors.

\section{Results and discussion}

\subsection{Gadolinium zirconate}

The heat capacity of $\mathrm{Gd}_{2} \mathrm{Zr}_{2} \mathrm{O}_{7}$ obtained by adiabatic calorimeter and PPMS measurements is plotted in figure 1 , where the inset of the figure shows the results below $T=25 \mathrm{~K}$. It exhibits a thermal anomaly below $T=15$ $\mathrm{K}$, which is related to the degeneracy of the ground state of the $\mathrm{Gd}^{3+}$ ion (see below). At low temperature but above the thermal anomaly temperature domain, the heat capacity has been fitted to the equation $C_{\mathrm{p}}=\alpha \cdot T^{3}$ (figure 2), yielding $C_{\mathrm{p}}^{\circ}\left(\mathrm{Gd}_{2} \mathrm{Zr}_{2} \mathrm{O}_{7}\right)=4.952 \cdot 10^{-4} \cdot T^{3}$.

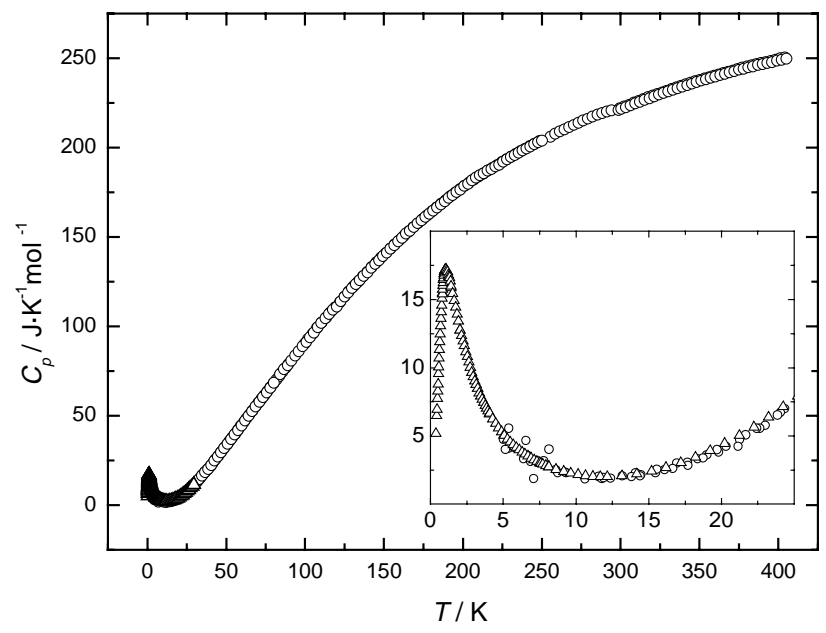

FIGURE 1 . Heat capacity of $\mathrm{Gd}_{2} \mathrm{Zr}_{2} \mathrm{O}_{7}$ measured by adiabatic calorimetry $(\bigcirc)$ and PPMS $(\triangle)$ plotted against temperature. The inset shows the low temperature measurements $T=(0$ to 25$) \mathrm{K}$.

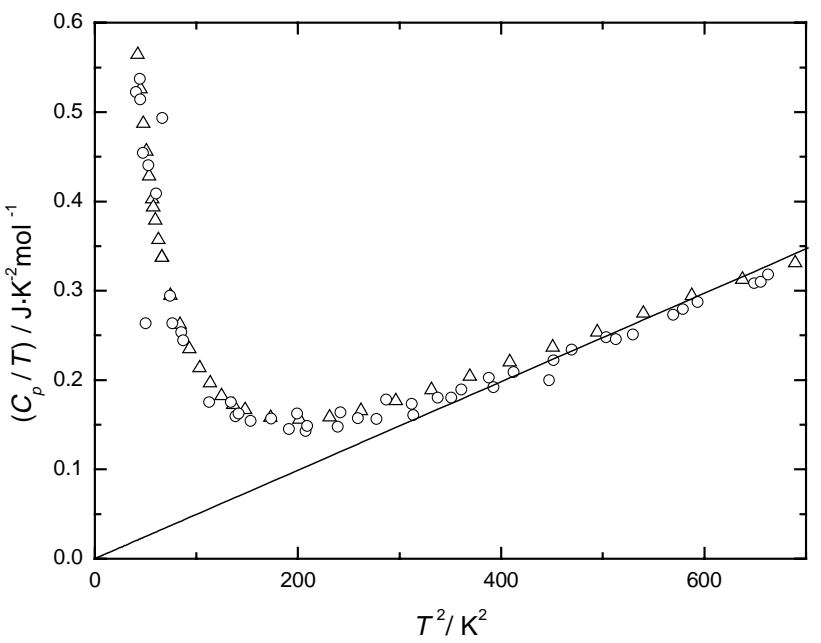

FIGURE 2. Low temperature heat capacity as $\left(C_{\mathrm{p}} / T\right)$ of $\mathrm{Gd}_{2} \mathrm{Zr}_{2} \mathrm{O}_{7}$ measured by adiabatic calorimetry $(O)$ and $\operatorname{PPMS}(\triangle)$ plotted against $T^{2}$; the solid line shows the $\alpha \cdot T^{3}$ fit as given in the text.

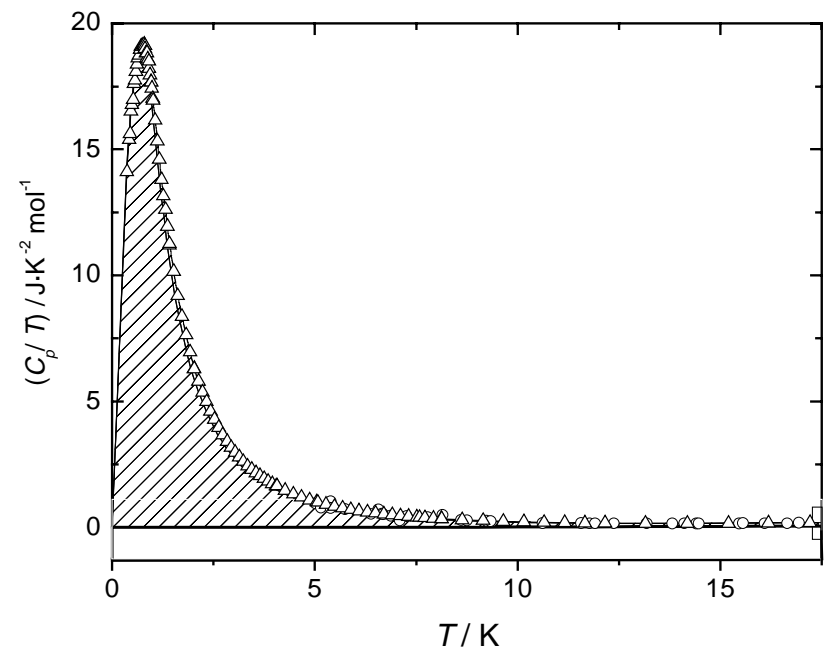

FIGURE 3. Low temperature heat capacity as $\left(C_{\mathrm{p}} / T\right)$ of $\mathrm{Gd}_{2} \mathrm{Zr}_{2} \mathrm{O}_{7}$ $(-)$ measured by adiabatic calorimetry $(O)$ and PPMS $(\triangle)$ and its lattice component (-) plotted against temperature. The dashed area corresponds to the Schottky component. 
From this equation, the heat capacity value at $T=20 \mathrm{~K}$ was obtained as $C_{\mathrm{p}}^{\circ}\left(\mathrm{Gd}_{2} \mathrm{Zr}_{2} \mathrm{O}_{7}, 20 \mathrm{~K}\right)=3.962 \mathrm{~J} \cdot \mathrm{K}^{-1}$. $\mathrm{mol}^{-1}$.

The heat capacity $C_{\mathrm{p}}$ as well as the entropy $S$ can be described as the sum of two components: the lattice contribution $C_{\text {lat }}$ or $S_{\text {lat }}$ due to the vibrations of the ions in the crystal and the excess or Schottky contribution $C_{\text {exs }}$ or $S_{\text {exs }}$ due to the electron population of the Stark levels, arising from splitting of the ground state due to the crystal field. In case of $\mathrm{Gd}_{2} \mathrm{Zr}_{2} \mathrm{O}_{7}$, in which the $\mathrm{Gd}$ ion has a half-filled $4 \mathrm{f}$-shell, there is no crystal-field effect since the orbital part of the angular momentum is zero (spectroscopic term ${ }^{8} \mathrm{~S}_{7 / 2}$ ). As a result the heat capacity

TABLE 3

Smoothed thermodynamic functions of $\mathrm{Gd}_{2} \mathrm{Zr}_{2} \mathrm{O}_{7}$ between $T=(20$ and 400$) \mathrm{K}\left(\Phi_{\mathrm{m}}^{\circ}(T)=-\left[\Delta G_{\mathrm{m}}^{\circ}(T)-\Delta H_{\mathrm{m}}^{\circ}(0 \mathrm{~K})\right] / T\right)$

\begin{tabular}{|c|c|c|c|c|}
\hline \multirow[t]{2}{*}{$T / \mathrm{K}$} & $C_{\mathrm{p}, \mathrm{m}}^{0}(T)$ & $S_{\mathrm{m}}^{\circ}(T)$ & $\Delta_{0}^{\mathrm{T}} H_{\mathrm{m}}(T)$ & $\Phi_{\mathrm{m}}^{\mathrm{o}}(T)$ \\
\hline & $\left(\mathrm{J} \cdot \mathrm{K}^{-1} \cdot \mathrm{mol}^{-1}\right)$ & $\left(\mathrm{J} \cdot \mathrm{K}^{-1} \cdot \mathrm{mol}^{-1}\right)$ & $\left(\mathrm{J} \cdot \mathrm{mol}^{-1}\right)$ & $\left(\mathrm{J} \cdot \mathrm{K}^{-1} \cdot \mathrm{mol}^{-1}\right)$ \\
\hline 20 & 3.962 & 36.75 & 88.72 & 32.3 \\
\hline 25 & 7.500 & 37.97 & 116.5 & 33.3 \\
\hline 30 & 11.48 & 39.67 & 165.6 & 34.2 \\
\hline 35 & 16.64 & 41.82 & 235.5 & 35.1 \\
\hline 40 & 21.62 & 44.37 & 331.1 & 36.1 \\
\hline 45 & 27.37 & 47.25 & 453.8 & 37.2 \\
\hline 50 & 33.19 & 50.43 & 604.9 & 38.3 \\
\hline 55 & 39.02 & 53.87 & 786.1 & 39.6 \\
\hline 60 & 44.79 & 57.51 & 995.8 & 40.9 \\
\hline 65 & 50.69 & 61.33 & 1234 & 42.4 \\
\hline 70 & 56.46 & 65.30 & 1502 & 43.8 \\
\hline 75 & 62.20 & 69.39 & 1799 & 45.4 \\
\hline 80 & 67.98 & 73.59 & 1617 & 47.0 \\
\hline 85 & 73.77 & 77.88 & 2477 & 48.7 \\
\hline 90 & 79.30 & 82.25 & 2859 & 50.5 \\
\hline 95 & 84.79 & 86.68 & 3271 & 52.3 \\
\hline 100 & 90.19 & 91.17 & 3708 & 54.1 \\
\hline 110 & 101.3 & 100.3 & 4662 & 57.9 \\
\hline 120 & 110.8 & 109.6 & 5731 & 61.8 \\
\hline 130 & 121.5 & 118.9 & 6894 & 65.8 \\
\hline 140 & 130.5 & 128.2 & 8149 & 70.0 \\
\hline 150 & 139.9 & 137.5 & 9510 & 74.1 \\
\hline 160 & 148.3 & 146.8 & 10,958 & 78.3 \\
\hline 170 & 156.3 & 156.1 & 12,457 & 82.8 \\
\hline 180 & 163.9 & 165.2 & 14,081 & 87.0 \\
\hline 190 & 171.1 & 174.3 & 15,754 & 91.3 \\
\hline 200 & 177.8 & 183.2 & 17,471 & 95.9 \\
\hline 210 & 183.8 & 192.0 & 19,311 & 100.1 \\
\hline 220 & 189.1 & 200.7 & 21,166 & 104.5 \\
\hline 230 & 194.3 & 209.2 & 23,064 & 108.9 \\
\hline 240 & 199.4 & 217.6 & 25,059 & 113.2 \\
\hline 250 & 203.6 & 225.8 & 27,064 & 117.6 \\
\hline 260 & 208.2 & 233.9 & 29,120 & 121.9 \\
\hline 270 & 212.4 & 241.8 & 31,223 & 126.2 \\
\hline 280 & 215.8 & 249.6 & 33,367 & 130.5 \\
\hline 290 & 219.2 & 257.3 & 35,548 & 134.7 \\
\hline 298.15 & 221.8 & 263.4 & 37,346 & 138.1 \\
\hline 300 & 222.4 & 264.8 & 37,755 & 138.9 \\
\hline 310 & 225.4 & 272.1 & 39,985 & 143.1 \\
\hline 320 & 228.1 & 279.3 & 42,250 & 147.3 \\
\hline 330 & 231.3 & 286.4 & 44,548 & 151.4 \\
\hline 340 & 234.2 & 293.3 & 46,875 & 155.4 \\
\hline 350 & 237.0 & 300.1 & 49,231 & 159.5 \\
\hline 360 & 239.7 & 306.9 & 51,615 & 163.5 \\
\hline 370 & 242.2 & 313.5 & 54,024 & 167.4 \\
\hline 380 & 244.6 & 320.0 & 56,458 & 171.4 \\
\hline 390 & 246.7 & 326.3 & 58,915 & 175.3 \\
\hline
\end{tabular}

measured corresponds to the lattice component, except in the temperature domain of the observed thermal anomaly (below $T=15 \mathrm{~K}$ ). Thus, in the low temperature domain, extrapolation of equation (1) to $T=0 \mathrm{~K}$ yields the lattice contribution to the heat capacity, giving $S_{\text {lat }}\left(\mathrm{Gd}_{2} \mathrm{Zr}_{2} \mathrm{O}_{7}, 20 \mathrm{~K}\right)=1.321 \mathrm{~J} \cdot \mathrm{K}^{-1} \cdot \mathrm{mol}^{-1}$ by integration of $C_{\mathrm{p}} / T$ from $T=(0$ to 20$) \mathrm{K}$.

From the difference between the experimental curve (triangles in figure 3) and the lattice component determined (thick solid line in figure 3 ), the entropy change of the anomaly is obtained as $S_{\mathrm{exs}}\left(\mathrm{Gd}_{2} \mathrm{Zr}_{2} \mathrm{O}_{7}\right)=35.430$ $\mathrm{J} \cdot \mathrm{K}^{-1} \cdot \mathrm{mol}^{-1}=1.025 \cdot(2 R \cdot \ln 8)$. This value is in excellent agreement with the theoretical value for a Schottky contribution arising from the ground-state degeneracy only, which is $R \cdot \ln \left(g_{0}\right)$ per mole of gadolinium. Since

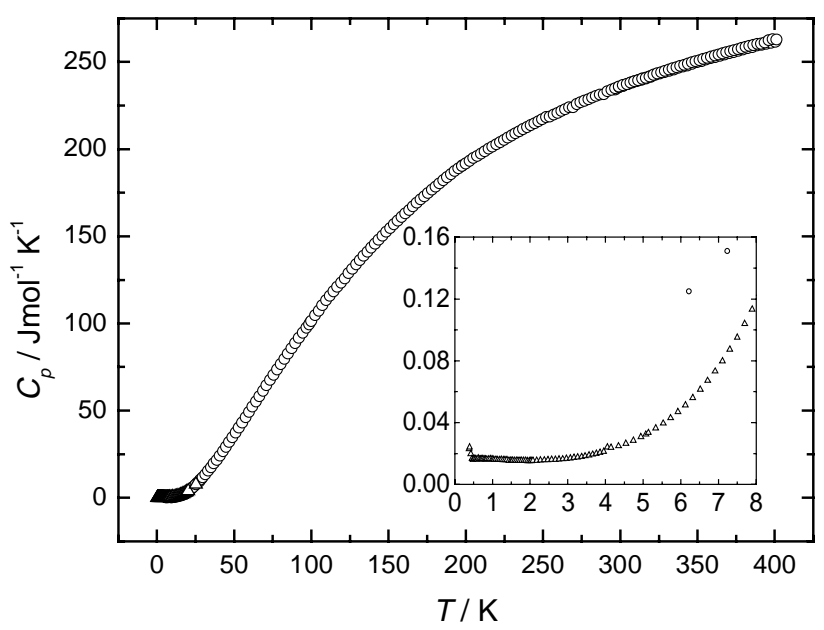

FIGURE 4. Heat capacity of $\mathrm{Eu}_{2} \mathrm{Zr}_{2} \mathrm{O}_{7}$ measured by adiabatic calorimetry $(O)$ and PPMS $(\triangle)$ plotted against temperature. The inset

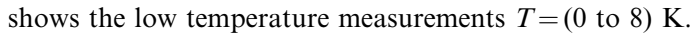

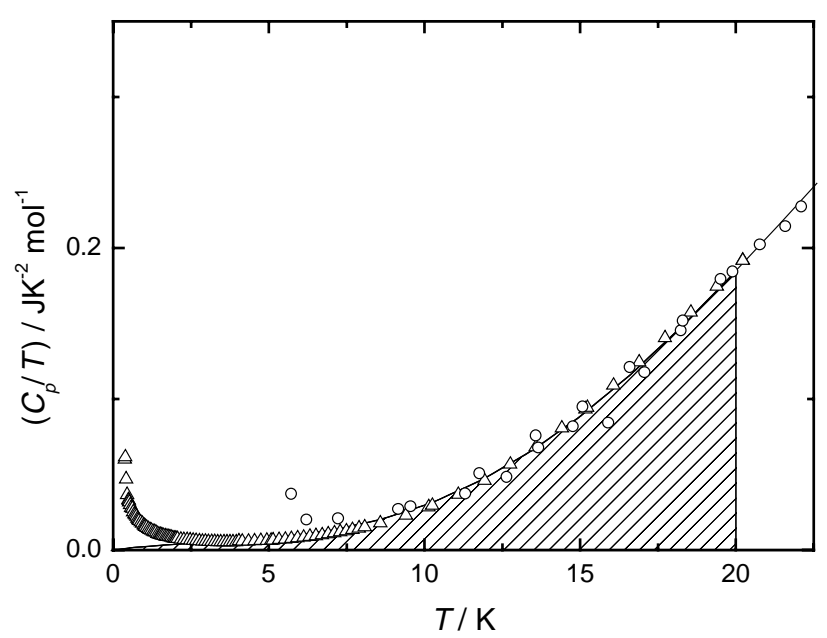

FIGURE 5. Low temperature heat capacity as $\left(C_{\mathrm{p}} / T\right)$ of $\mathrm{Eu}_{2} \mathrm{Zr}_{2} \mathrm{O}_{7}$ measured by adiabatic calorimetry $(\bigcirc)$ and $\operatorname{PPMS}(\triangle)$ plotted against temperature, and the interpolation to $T=0 \mathrm{~K}$. The dashed area corresponds to the entropy at $T=20 \mathrm{~K}$. 
the ground multiplet is ${ }^{8} \mathrm{~S}_{7 / 2}$, the degeneracy is $g_{0}=8$. The total entropy can then be obtained from the sum of the lattice and the Schottky component, yielding $S^{\circ}\left(\mathrm{Gd}_{2} \mathrm{Zr}_{2} \mathrm{O}_{7}, 20 \mathrm{~K}\right)=36.751 \mathrm{~J} \cdot \mathrm{K}^{-1} \cdot \mathrm{mol}^{-1}$. Using the heat capacity and the entropy at $T=20 \mathrm{~K}$, the smoothed thermodynamic functions of $\mathrm{Gd}_{2} \mathrm{Zr}_{2} \mathrm{O}_{7}$ were derived in the temperature range ( 20 to 400$) \mathrm{K}$ and are summarised in table 3 .

\subsection{Europium zirconate}

The heat capacity data obtained for $\mathrm{Eu}_{2} \mathrm{Zr}_{2} \mathrm{O}_{7}$ by adiabatic calorimeter and PPMS measurements are

TABLE 4

Smoothed thermodynamic functions of $\mathrm{Eu}_{2} \mathrm{Zr}_{2} \mathrm{O}_{7}$ between $T=(20$ and $400) \mathrm{K}\left(\Phi_{\mathrm{m}}^{\circ}(T)=-\left[\Delta G_{\mathrm{m}}^{\circ}(T)-\Delta H_{\mathrm{m}}^{\circ}(0 \mathrm{~K})\right] / T\right)$

\begin{tabular}{|c|c|c|c|c|}
\hline \multirow[t]{2}{*}{$T / \mathrm{K}$} & $C_{\mathrm{p}, \mathrm{m}}^{0}(T)$ & $S_{\mathrm{m}}^{\circ}(T)$ & $\Delta_{0}^{\mathrm{T}} H_{\mathrm{m}}(T)$ & $\Phi_{\mathrm{m}}^{\mathrm{o}}(T)$ \\
\hline & $\left(\mathrm{J} \cdot \mathrm{K}^{-1} \cdot \mathrm{mol}^{-1}\right)$ & $\overline{\left(\mathrm{J} \cdot \mathrm{K}^{-1} \cdot \mathrm{mol}^{-1}\right)}$ & $\left(\mathrm{J} \cdot \mathrm{mol}^{-1}\right)$ & $\left(\mathrm{J} \cdot \mathrm{K}^{-1} \cdot \mathrm{mol}^{-1}\right)$ \\
\hline 20 & 3.697 & 1.055 & 15.98 & 0.256 \\
\hline 25 & 7.462 & 2.050 & 42.81 & 0.337 \\
\hline 30 & 12.05 & 3.813 & 92.05 & 0.744 \\
\hline 35 & 17.44 & 6.068 & 165.2 & 1.349 \\
\hline 40 & 23.12 & 8.773 & 267.0 & 2.099 \\
\hline 45 & 29.53 & 11.87 & 398.7 & 3.009 \\
\hline 50 & 36.16 & 15.32 & 563.0 & 4.064 \\
\hline 55 & 42.81 & 19.08 & 760.5 & 5.255 \\
\hline 60 & 49.68 & 23.10 & 992 & 6.572 \\
\hline 65 & 56.43 & 27.34 & 1257 & 8.006 \\
\hline 70 & 63.22 & 31.77 & 1556 & 9.544 \\
\hline 75 & 69.88 & 36.36 & 1889 & 11.18 \\
\hline 80 & 76.44 & 41.08 & 2255 & 12.90 \\
\hline 85 & 82.96 & 45.91 & 2653 & 14.70 \\
\hline 90 & 89.28 & 50.83 & 3084 & 16.57 \\
\hline 95 & 95.46 & 55.83 & 3546 & 18.50 \\
\hline 100 & 101.5 & 60.88 & 4041 & 20.47 \\
\hline 110 & 113.8 & 71.14 & 5118 & 24.61 \\
\hline 120 & 124.1 & 81.49 & 6309 & 28.92 \\
\hline 130 & 135.0 & 91.86 & 7605 & 33.36 \\
\hline 140 & 144.7 & 102.2 & 9004 & 37.91 \\
\hline 150 & 153.9 & 112.5 & 10,497 & 42.55 \\
\hline 160 & 162.4 & 122.7 & 12,079 & 47.24 \\
\hline 170 & 170.7 & 132.8 & 13,745 & 51.98 \\
\hline 180 & 178.2 & 142.8 & 15,489 & 56.75 \\
\hline 190 & 185.4 & 152.6 & 17,307 & 61.54 \\
\hline 200 & 191.8 & 162.3 & 19,194 & 66.33 \\
\hline 210 & 197.5 & 171.8 & 21,142 & 71.13 \\
\hline 220 & 202.9 & 181.1 & 23,145 & 75.92 \\
\hline 230 & 208.0 & 190.3 & 25,199 & 80.69 \\
\hline 240 & 212.8 & 199.2 & 27,303 & 85.45 \\
\hline 250 & 217.3 & 208.0 & 29,454 & 90.17 \\
\hline 260 & 221.1 & 216.6 & 31,645 & 94.87 \\
\hline 270 & 225.2 & 225.0 & 33,877 & 99.54 \\
\hline 280 & 228.9 & 233.3 & 36,147 & 104.2 \\
\hline 290 & 231.9 & 241.4 & 38,453 & 108.8 \\
\hline 298.15 & 235.4 & 247.8 & 40,359 & 112.5 \\
\hline 300 & 235.7 & 249.3 & 40,794 & 113.3 \\
\hline 310 & 239.0 & 257.1 & 43,168 & 117.8 \\
\hline 320 & 242.1 & 264.7 & 45,576 & 122.3 \\
\hline 330 & 245.2 & 272.2 & 48,013 & 126.7 \\
\hline 340 & 248.0 & 279.6 & 50,479 & 131.1 \\
\hline 350 & 250.7 & 286.8 & 52,973 & 135.5 \\
\hline 360 & 253.4 & 293.9 & 55,493 & 139.8 \\
\hline 370 & 255.8 & 300.9 & 58,038 & 144.0 \\
\hline 380 & 258.3 & 307.7 & 60,609 & 148.2 \\
\hline 390 & 260.4 & 314.5 & 63,203 & 152.4 \\
\hline
\end{tabular}

plotted in figure 4 where the inset of the figure shows the low temperature results (below $T=8 \mathrm{~K}$ ). Below $T=8$ $\mathrm{K}$, the adiabatic data are scattered, which is due to the fact that the apparatus was operated at its lower temperature limit.

No thermal anomaly is expected for this compound as the ground term is ${ }^{7} \mathrm{~F}_{0}$ and its degeneracy $g_{0}=1$. However, a small anomalous tendency is visible below $T=1 \mathrm{~K}$. This could be due to the presence of $\mathrm{Gd}^{3+}$ or $\mathrm{Eu}^{2+}$ impurity $\left(g_{0}=8\right)$. The analysis report of the europium nitrate precursor mentioned $10 \cdot 10^{-6}$ gadolinium impurity and, as the compound was sintered in air, $\mathrm{Eu}^{2+}$ is not expected. Since the thermal anomaly observed for $\mathrm{Gd}^{3+}$ is so intense that even a small quantity of $\mathrm{Gd}^{3+}$ would be detected, the anomalous behaviour in $\mathrm{Eu}_{2} \mathrm{Zr}_{2} \mathrm{O}_{7}$ is assigned to this impurity and will be neglected for the further analysis.

From data measured in the temperature range (3 to 20) $\mathrm{K}$ the heat capacity of $\mathrm{Eu}_{2} \mathrm{Zr}_{2} \mathrm{O}_{7}$ has been extrapolated to $T=0 \mathrm{~K}$, as shown in figure 5 . From this curve, the heat capacity has been determined at $T=20 \mathrm{~K}$, yielding $C_{\mathrm{p}}^{\circ}\left(\mathrm{Eu}_{2} \mathrm{Zr}_{2} \mathrm{O}_{7}, 20 \mathrm{~K}\right)=3.697 \mathrm{~J} \cdot \mathrm{K}^{-1} \cdot \mathrm{mol}^{-1}$. The entropy at the same temperature has been determined as the surface under the curve between $T=(0$ and 20$) \mathrm{K}$, as shown by the dashed surface in figure 5 , yielding $S^{\circ}\left(\mathrm{Eu}_{2} \mathrm{Zr}_{2}\right.$ $\left.\mathrm{O}_{7}, 20 \mathrm{~K}\right)=1.055 \mathrm{~J} \cdot \mathrm{K}^{-1} \cdot \mathrm{mol}^{-1}$. Using the heat capacity and the entropy at $T=20 \mathrm{~K}$, the thermodynamic functions of $\mathrm{Eu}_{2} \mathrm{Zr}_{2} \mathrm{O}_{7}$ were derived in the temperature range (20 to 400$) \mathrm{K}$ and are summarised in table 4 .

\section{Discussion}

The heat capacity of $\mathrm{Gd}_{2} \mathrm{Zr}_{2} \mathrm{O}_{7}$ and $\mathrm{Eu}_{2} \mathrm{Zr}_{2} \mathrm{O}_{7}$ are compared in figure 6 to the results previously obtained for $\mathrm{Nd}_{2} \mathrm{Zr}_{2} \mathrm{O}_{7}$ by the present authors [28] and for

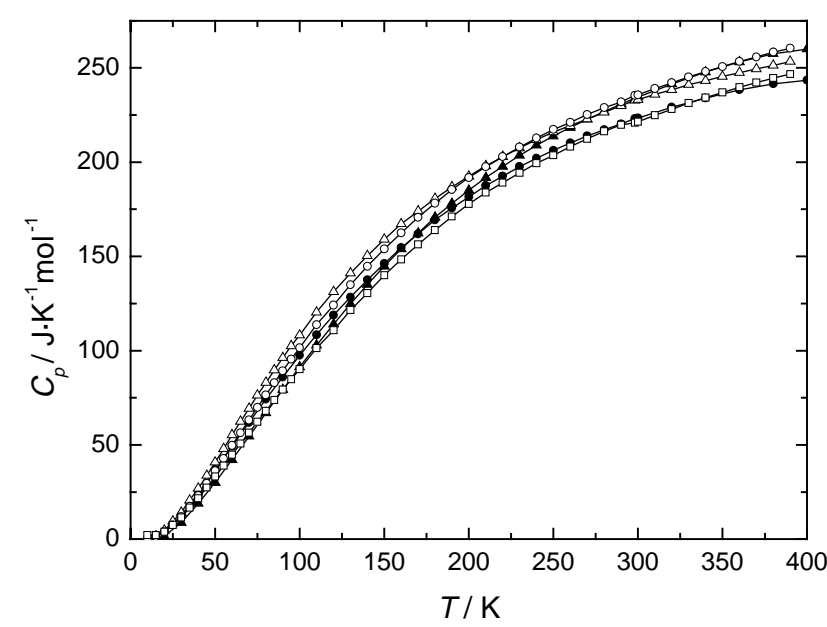

FIGURE 6. Heat capacity obtained for $\mathrm{Gd}_{2} \mathrm{Zr}_{2} \mathrm{O}_{7}(\square)$ and $\mathrm{Eu}_{2} \mathrm{Zr}_{2} \mathrm{O}_{7}$ (O) plotted against temperature compared to the results reported for $\mathrm{Nd}_{2} \mathrm{Zr}_{2} \mathrm{O}_{7}(\triangle)$ by the present authors [28] and for $\mathrm{La}_{2} \mathrm{Zr}_{2} \mathrm{O}_{7}(\bullet)$ and $\mathrm{Ce}_{2} \mathrm{Zr}_{2} \mathrm{O}_{7}(\boldsymbol{\Delta})$ by Bolech et al. [24,25]. 

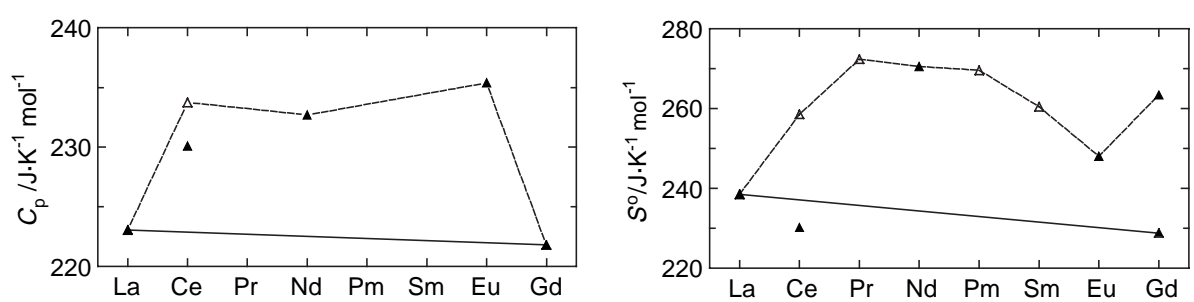

FIGURE 7. Heat capacity and entropy of the lanthanide zirconate $\mathrm{Ln}_{2} \mathrm{Zr}_{2} \mathrm{O}_{7}$ for $\mathrm{Ln}=\mathrm{La}$ to $\mathrm{Gd}$ at the standard temperature $T=298.15 \mathrm{~K}$. Closed symbols ( $\boldsymbol{\Delta})$ indicate the measured values, and the lattice component interpolated from the $\mathrm{La}_{2} \mathrm{Zr}_{2} \mathrm{O}_{7}$ and $\mathrm{Gd}_{2} \mathrm{Zr}_{2} \mathrm{O}_{7}$ values is shown as a solid line. The open symbols $(\triangle)$ show the values estimated from the ground-state degeneracy (see detail in Section 4), except for $\mathrm{Ce}_{2} \mathrm{Zr}_{2} \mathrm{O}_{7}$ which have been calculated from the crystal field energies.
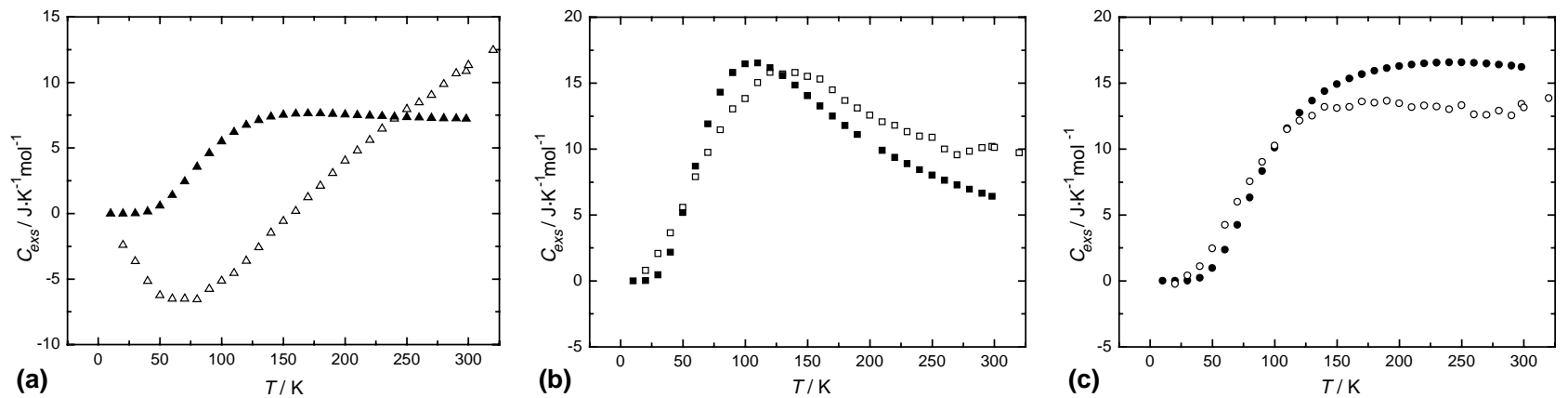

FIGURE 8. The excess (Schottky) heat capacity of: (a) $\mathrm{Ce}_{2} \mathrm{Zr}_{2} \mathrm{O}_{7}(\triangle)$, (b) $\mathrm{Nd}_{2} \mathrm{Zr}_{2} \mathrm{O}_{7}$ ( $\square$ ) and (c) $\mathrm{Eu}_{2} \mathrm{Zr}_{2} \mathrm{O}_{7}$ (O). Open symbols correspond to the experimental values and closed symbols to values calculated from the energy level according to equation (1).

$\mathrm{La}_{2} \mathrm{Zr}_{2} \mathrm{O}_{7}$ and $\mathrm{Ce}_{2} \mathrm{Zr}_{2} \mathrm{O}_{7}$ by Bolech et al. [24,25]. The heat capacity curves of $\mathrm{La}_{2} \mathrm{Zr}_{2} \mathrm{O}_{7}$ and $\mathrm{Gd}_{2} \mathrm{Zr}_{2} \mathrm{O}_{7}$ are almost identical above $T=20 \mathrm{~K}$. The curves of $\mathrm{Eu}_{2}$ $\mathrm{Zr}_{2} \mathrm{O}_{7}$ and $\mathrm{Nd}_{2} \mathrm{Zr}_{2} \mathrm{O}_{7}$ have the same shape but are slightly different, whereas the curve of $\mathrm{Ce}_{2} \mathrm{Zr}_{2} \mathrm{O}_{7}$ is the lowest at low temperature and the highest at high temperature. On first sight these differences can be explained by the effect of the Schottky contribution.

The excess or Schottky contribution can be calculated from the crystal field energies according to the following equations where $Q$ is the partitioning function described by the Maxwell-Boltzmann distribution law in equation (3), $T$ the temperature, $R$ the universal gas constant, $E_{i}$ the energy of the level $i$, and $g_{i}$, its degeneracy

$$
\begin{aligned}
C_{\mathrm{exs}}= & Q^{-2} \cdot R^{-2} \cdot T^{-2} \cdot\left[Q \cdot \sum_{i=1}^{n} g_{i} \cdot E_{i}^{2} \cdot \exp \left(-E_{i} / R \cdot T\right)-\right. \\
& \left.\left\{\sum_{i=1}^{n} g_{i} \cdot E_{i} \cdot \exp \left(-E_{i} / R \cdot T\right)\right\}^{2}\right], \\
S_{\mathrm{exs}}= & R \cdot \ln \left(g_{o}\right)+R \cdot \ln \left\{\sum_{i=1}^{n} g_{i} \cdot \exp \left(-E_{i} / R \cdot T\right)\right\}
\end{aligned}
$$

$Q=\sum_{i=0}^{n} g_{i} \cdot \exp \left(-E_{i} / R \cdot T\right)$

\begin{tabular}{|c|c|c|c|}
\hline & {$[\mathrm{SL}] \mathrm{J}$-state } & Degeneracy & $\operatorname{Energy}^{a}(h c)^{-1} / \mathrm{cm}^{-1}$ \\
\hline \multirow[t]{7}{*}{$\mathrm{Ce}_{2} \mathrm{Zr}_{2} \mathrm{O}_{7}$} & \multirow[t]{3}{*}{${ }^{2} \mathrm{~F}_{5 / 2}$} & 2 & 0 \\
\hline & & 2 & 253 \\
\hline & & 2 & 875 \\
\hline & \multirow[t]{4}{*}{${ }^{2} \mathrm{~F}_{7 / 2}$} & 2 & 2123 \\
\hline & & 2 & 2630 \\
\hline & & 2 & 2716 \\
\hline & & 2 & 3659 \\
\hline \multirow[t]{11}{*}{$\mathrm{Nd}_{2} \mathrm{Zr}_{2} \mathrm{O}_{7}$} & \multirow[t]{5}{*}{${ }^{4} \mathbf{I}_{9 / 2}$} & 2 & 0 \\
\hline & & 2 & 164 \\
\hline & & 2 & 224 \\
\hline & & 2 & 234 \\
\hline & & 2 & 590 \\
\hline & \multirow[t]{6}{*}{${ }^{4} \mathbf{I}_{11 / 2}$} & 2 & 1900 \\
\hline & & 2 & 2027 \\
\hline & & 2 & 2052 \\
\hline & & 2 & 2085 \\
\hline & & 2 & 2251 \\
\hline & & 2 & 2263 \\
\hline \multirow[t]{11}{*}{$\mathrm{Eu}_{2} \mathrm{Zr}_{2} \mathrm{O}_{7}$} & ${ }^{7} \mathrm{~F}_{0}$ & 1 & 0 \\
\hline & \multirow[t]{2}{*}{${ }^{7} \mathrm{~F}_{1}$} & 2 & 264 \\
\hline & & 1 & 612 \\
\hline & \multirow[t]{3}{*}{${ }^{7} \mathrm{~F}_{2}$} & 2 & 762 \\
\hline & & 1 & 880 \\
\hline & & 2 & 1452 \\
\hline & \multirow[t]{5}{*}{${ }^{7} F_{3}$} & 1 & 1714 \\
\hline & & 1 & 1776 \\
\hline & & 2 & 1965 \\
\hline & & 2 & 2145 \\
\hline & & 1 & 2236 \\
\hline
\end{tabular}

TABLE 5

Energy levels of the $\mathrm{Ln}^{3+}$ cations in the pyrochlore structure $\mathrm{Ln}_{2} \mathrm{Zr}_{2} \mathrm{O}_{7}$

${ }^{a} h$ is Planck constant and $c$ is the speed of light in vacuum. 
In general, the lattice heat capacity of isostructural lanthanide compounds are close to each other and linearly decrease along the series [30]. In the temperature range between $T=(20$ and 300$) \mathrm{K}$ the Schottky contributions to the heat capacity are zero for the La and Gd compounds as their lanthanide f-shell are empty and half-filled, respectively. The excess contribution to the entropy is also zero for $\mathrm{La}_{2} \mathrm{Zr}_{2} \mathrm{O}_{7}$ and is only composed of the ground level contribution for $\mathrm{Gd}_{2} \mathrm{Zr}_{2} \mathrm{O}_{7}$. From these statements, the lattice component of the heat capacity and of the entropy can be interpolated from the $\mathrm{La}_{2} \mathrm{Zr}_{2} \mathrm{O}_{7}$ and $\mathrm{Gd}_{2} \mathrm{Zr}_{2} \mathrm{O}_{7}$ values for the complete lanthanide series (with the value $2 R \cdot \ln 8$ subtracted from the $\mathrm{Gd}_{2} \mathrm{Zr}_{2} \mathrm{O}_{7}$ entropy data). These interpolations are shown for $T=298.15 \mathrm{~K}$ in figure 7 and have been reproduced for the complete temperature range (20 to 400) K.

By subtracting these lattice heat capacity values from the measured data, the Schottky heat capacity has been

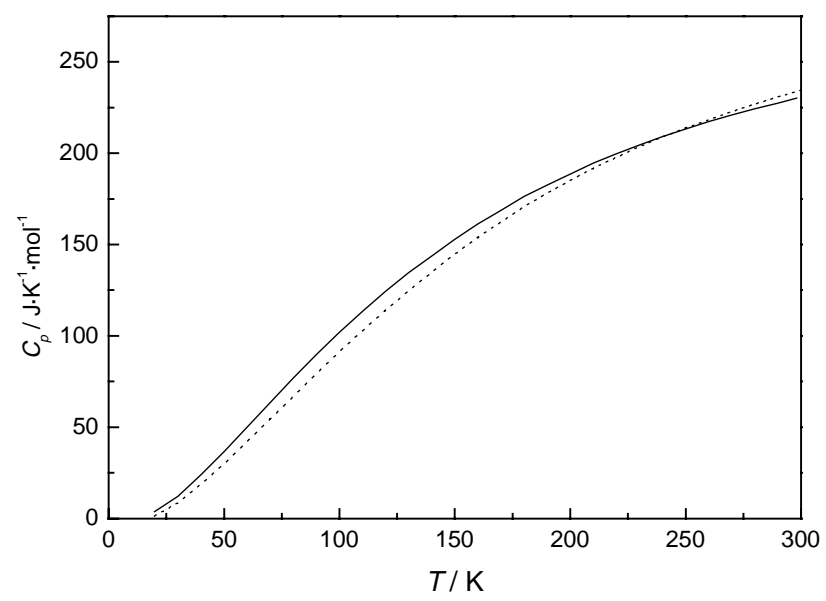

FIGURE 9. Calculated (-) and experimental ( . . $[24,25]$ heat capacity of $\mathrm{Ce}_{2} \mathrm{Zr}_{2} \mathrm{O}_{7}$ plotted against the temperature. determined. The resulting values are plotted as open symbols in figure 8 . The excess contribution to the heat capacity of $\mathrm{Ce}_{2} \mathrm{Zr}_{2} \mathrm{O}_{7}$ is negative between $T=(20$ and 150) $\mathrm{K}$ which is not in agreement with the expected behaviour. The significantly lower heat capacity of $\mathrm{Ce}_{2} \mathrm{Zr}_{2} \mathrm{O}_{7}$ compared to $\mathrm{La}_{2} \mathrm{Zr}_{2} \mathrm{O}_{7}$ was explained by Bolech as the result of lattice defects introduced by the sintering. However, it can also be an effect of a partial oxidation of the cerium into $\mathrm{Ce}^{4+}$.

The Schottky contributions of the zirconates have also been calculated from the crystal field energies of the lanthanide ions in the pyrochlore structure. The electronic energy levels were obtained from optical spectroscopy measurements on $\mathrm{La}_{2} \mathrm{Zr}_{2} \mathrm{O}_{7}$ doped with $5 \%$ $\mathrm{Eu}$, scaled using the crystal field parameters of $\mathrm{Ho}_{2} \mathrm{Ti}_{2} \mathrm{O}_{7}$ [31]. The resulting energy levels are summarised in table 5. The Schottky contributions to the heat capacity thus obtained are plotted as closed symbols in figure 8. Except for $\mathrm{Ce}_{2} \mathrm{Zr}_{2} \mathrm{O}_{7}$ these data agree well with those determined from the experimental heat capacity data. The shapes of the experimental and calculated curves are similar, but do not fully overlap, indicating that the crystal field energies need further refining.

The total heat capacity of $\mathrm{Ce}_{2} \mathrm{Zr}_{2} \mathrm{O}_{7}$ has been simulated from the lattice components determined by interpolation and from the Schottky contributions calculated from the crystal field energies. The results are plotted in figure 9. Table 6 gives the resulting entropy value at $T=298.15 \mathrm{~K}$, which is $28.3 \mathrm{~J} \cdot \mathrm{K}^{-1} \cdot \mathrm{mol}^{-1}$ higher than the result of Bolech et al. [24,25]. In this table, the entropies of the other lanthanide pyrochlore zirconates are estimated, from the lattice component and the excess contribution calculated from the degeneracy of the lowest [SL]J-state, neglecting crystal field splitting. This may lead to a small overestimation of the entropies of up to $4 \mathrm{~J} \cdot \mathrm{K}^{-1} \cdot \mathrm{mol}^{-1}$.

TABLE 6

The standard entropies (at $T=298.15 \mathrm{~K}$ ) of the lanthanide pyrochlores $\mathrm{Ln}_{2} \mathrm{Zr}_{2} \mathrm{O}_{7}$ obtained from the lattice and excess components $\left(S_{\text {tot }}=S_{\text {lat }}+2 S_{\text {exs }}\right)$

\begin{tabular}{|c|c|c|c|c|}
\hline & $S_{\text {lat }}$ & $S_{\mathrm{exs}}$ & $S_{\mathrm{tot}}$ & $S_{\text {exp }}$ \\
\hline & $\left(\mathrm{J} \cdot \mathrm{K}^{-1} \cdot \mathrm{mol}^{-1}\right)$ & $\left(\mathrm{J} \cdot \mathrm{K}^{-1} \cdot \mathrm{mol}^{-1}\right)$ & $\left(\mathrm{J} \cdot \mathrm{K}^{-1} \cdot \mathrm{mol}^{-1}\right)$ & $\left(\mathrm{J} \cdot \mathrm{K}^{-1} \cdot \mathrm{mol}^{-1}\right)$ \\
\hline $\mathrm{La}_{2} \mathrm{Zr}_{2} \mathrm{O}_{7}$ & 238.5 & 0.00 & 238.5 & $238.5 \pm 0.5^{a}$ \\
\hline $\mathrm{Ce}_{2} \mathrm{Zr}_{2} \mathrm{O}_{7}$ & 237.1 & 10.69 & 258.5 & $230.2 \pm 0.5^{a}$ \\
\hline $\mathrm{Pr}_{2} \mathrm{Zr}_{2} \mathrm{O}_{7}$ & 235.8 & 18.27 & 272.3 & \\
\hline $\mathrm{Nd}_{2} \mathrm{Zr}_{2} \mathrm{O}_{7}$ & 234.4 & 17.13 & 268.6 & $270.5 \pm 0.6^{b}$ \\
\hline $\mathrm{Pm}_{2} \mathrm{Zr}_{2} \mathrm{O}_{7}$ & 233.0 & 18.27 & 269.5 & \\
\hline $\mathrm{Sm}_{2} \mathrm{Zr}_{2} \mathrm{O}_{7}$ & 231.6 & 14.42 & 260.4 & \\
\hline $\mathrm{Eu}_{2} \mathrm{Zr}_{2} \mathrm{O}_{7}$ & 230.2 & 9.92 & 250.01 & $247.8 \pm 0.5^{c}$ \\
\hline $\mathrm{Gd}_{2} \mathrm{Zr}_{2} \mathrm{O}_{7}$ & 228.8 & 17.29 & 263.4 & $263.4 \pm 0.5^{c}$ \\
\hline
\end{tabular}

When the excess values are given in italic numbers, the value has been obtained from the unsplit lowest [SL]J-state.

${ }^{a}$ Bolech et al. [24].

${ }^{b}$ Lutique et al. [28]; note that the correct value at $T=298.15 \mathrm{~K}$ is $S_{\mathrm{m}}^{\circ}=(270.5 \pm 0.6) \mathrm{J} \cdot \mathrm{K}^{-1} \cdot \mathrm{mol}^{-1}$ as given in table 3 of [28] and not (264.1 \pm 0.6$)$

$\mathrm{J} \cdot \mathrm{K}^{-1} \cdot \mathrm{mol}^{-1}$ as given in the text of this reference (which is the value at $T=290 \mathrm{~K}$ ).

${ }^{c}$ This study. 


\section{Conclusions}

The thermodynamic functions of $\mathrm{Gd}_{2} \mathrm{Zr}_{2} \mathrm{O}_{7}$ and $\mathrm{Eu}_{2}-$ $\mathrm{Zr}_{2} \mathrm{O}_{7}$ were determined in the temperature range (20 to 400) $\mathrm{K}$ from heat capacity measurements. Their heat capacity and entropy were compared with those of $\mathrm{Nd}_{2} \mathrm{Zr}_{2} \mathrm{O}_{7}, \mathrm{La}_{2} \mathrm{Zr}_{2} \mathrm{O}_{7}$ and $\mathrm{Ce}_{2} \mathrm{Zr}_{2} \mathrm{O}_{7}$ available in the literature. From the results the lattice heat capacity was determined by interpolation between $\mathrm{La}_{2} \mathrm{Zr}_{2} \mathrm{O}_{7}$ and $\mathrm{Gd}_{2} \mathrm{Zr}_{2} \mathrm{O}_{7}$ and the Schottky contribution was determined by subtracting the lattice heat capacity from the experimental data. These data were compared with values calculated from crystal field energies. Except for $\mathrm{Ce}_{2} \mathrm{Zr}_{2} \mathrm{O}_{7}$, both sets of data are coherent. The total heat capacity and entropy of this compound has been deduced for the other lanthanide pyrochlores from the calculated lattice and Schottky contributions.

\section{Acknowledgements}

S.L. and P.J. acknowledge the European Commission for support given in the frame of the program "training and mobility of researchers".

\section{References}

[1] A.P. Ramirez, A. Hayashi, R.J. Cava, R. Siddharthan, B.S. Shastry, Nature 399 (1999) 333-335.

[2] P. Bonville, J.A. Hodges, M. Ocio, J.P. Sanchez, P. Vulliet, S. Sosin, D. Braithwaite, J. Phys. Condens. Matter 15 (2003) 7777 7778.

[3] R. Higashinaka, H. Ukazawa, Y. Maeno, Physica B 329-333 (2003) $1040-1041$.

[4] R. Vassen, X.Q. Cao, D. Basu, D. Stöver, J. Am. Ceram. Soc. 83 (2000) 2023-2028.

[5] J. Wu, X. Wei, N.P. Padture, P.G. Klemens, M. Gell, E. Garcia, P. Miranzo, M.I. Osendi, J. Am. Ceram. Soc. 85 (2002) 30313035.

[6] X.Q. Cao, R. Vassen, W. Jungen, S. Schwartz, F. Tietz, D. Stöver, J. Am. Ceram. Soc. 84 (2001) 2086-2090.

[7] N.P. Padture et al., Acta Mater. 49 (2001) 2251-2257.

[8] T. Omata, H. Kishimoto, S. Otsuka-Yao-Matsuo, N. Ohtori, N. Umesaki, J. Solid-State Chem. 147 (1999) 573-583.

[9] N. Izu, T. Omata, S. Otsuka-Yao-Matsuo, J. Alloys Comp. 270 (1998) 107-114.

[10] S. Otsuka-Yao-Matsuo, T. Omata, N. Izu, H. Kishimoto, J. SolidState Chem. 138 (1998) 47-54.
[11] S. Otsuka-Yao-Matsuo, N. Izu, T. Omata, K. Ikeda, J. Electrochem. Soc. 145 (1998) 1406-1413.

[12] P.E. Raison, R.G. Haire, in: Proceeding GLOBAL'01, 9-13 Sept. 2001, Paris (on cd-rom).

[13] P.E. Raison, R.G. Haire, Z. Assefa, J. Nucl. Sci. Technol. (Suppl. 3) (2002) 725-728.

[14] S. Lutique, R.J.M. Konings, V.V. Rondinella, J. Somers, D. Staicu, T. Wiss, J. Nucl. Mater. 319 (2003) 59-64.

[15] S. Lutique, R.J.M. Konings, V.V. Rondinella, J. Somers, T. Wiss, in: CIMTEC 2002 - 10th International Ceramic Congress - Part D, 15-18 July 2002, Florence, Italy; Advances in Science and Technology, Vincenzini. P., pp. 241-248.

[16] S. Lutique, R.J.M. Konings, V.V. Rondinella, J. Somers, T. Wiss, J Alloys Comp. 352 (2003) 1-5.

[17] B.D. Begg, W.J. Weber, R. Devanathan, J.P. Icenhower, S. Thevuthasan, B. P. McGrail, Heavy-ion irradiation effects in pyrochlores, in: Environmental Issues and Waste Management Technologies V, 2000, pp. 553-560.

[18] B.D. Begg, N.J. Hess, W.J. Weber, R. Devanathan, J.P. Icenhower, S. Thevuthasan, B.P. McGrail, J. Nucl. Mater. 288 (2001) 208-216.

[19] S.X. Wang, B.D. Begg, L.M. Wang, R.C. Ewing, W.J. Weber, K.V. Godivan Kutty, J. Mater. Res. 14 (1999) 4470-4473.

[20] W.J. Weber, J.M. Wald, H. Matzke, Mater. Lett. 3 (1985) 173 180.

[21] J.M. Wald, P. Offermann, A study of radiation effects in curiumdoped $\mathrm{Gd}_{2} \mathrm{Ti}_{2} \mathrm{O}_{7}$ (pyrochlore) and $\mathrm{CaZrTi}_{2} \mathrm{O}_{7}$ (zirconolite), in: W. Lutze (Ed.), Scientific Basis for Radioactive Waste Management XII, 1982, pp. 369-378.

[22] V.R. Korneev, V.B. Glushkova, E.K. Keler, Izv. Akad. Nauk. SSSR Neorg. Materialy 7 (1971) 886-887.

[23] M. Bolech, E.H.P. Cordfunke, F.J.J.G. Janssen, A. Navrotsky, J. Am. Ceram. Soc. 78 (1995) 2257-2258.

[24] M. Bolech, E.H.P. Cordfunke, A.C.G. van Genderen, R.R. van der Laan, F.J.J.G. Janssen, J.C. Van Miltenburg, J. Phys. Chem. Solids 58 (1997) 433-439.

[25] M. Bolech, Ph.D. Thesis, University of Amsterdam, The Netherlands, 1998.

[26] K.B. Helean, B.D. Begg, A. Navrotsky, B. Ebbinghaus, W.J. Weber, R.C. Ewing, Mater. Res. Soc. Symp. Proc. 663 (2001) 691697.

[27] S. Lutique, Ph.D. Thesis, Université de Paris-Sud, U.F.R. Scientifique d'Orsay, France, 2003.

[28] S. Lutique, P. Javorskỳ, R.J.M. Konings, J.C. van Miltenburg, A.C.G. van Genderen, F. Wastin, J. Chem. Thermodyn. 35 (2003) 955-965.

[29] J.C. van Miltenburg, G.J.K. van den Berg, M.J. van Bommel, J. Chem. Thermodyn. 19 (1987) 1129-1138.

[30] R.J.M. Konings, J. Nucl. Mater. 295 (2001) 57-63.

[31] S. Rosenkranz, A.P. Ramirez, A. Hayashi, R.J. Cava, R. Siddharthan, B.S. Shastry, J. Appl. Phys. 87 (2000) 5914 5916.

JCT $04 / 26$ 